\title{
The Evolution of Internet Public Opinion in Emergent Event with Agent- Based Modeling and Simulation
}

\author{
Song Jiang* and $\mathrm{Wu}$ Peng
}

School of Economics and Management, Nanjing University of Science and Technology, Nanjing, Jiangsu, 210094, P.R. China

\begin{abstract}
In this paper, several factors are analyzed in the evolution of Internet public opinion in emergent events. The analysis was conducted with an illustration about the information spreading in "H7N9 Event" with the help of NetLogo. After agent-based modeling and simulation, some interesting results have been found: (1) compared to the Internet public opinions without government intervention, the situation with government interventions had a shorter time to reach stable state; (2) compared to the Internet public opinions with lower credibility of the government, the situation with higher credibility of the government had a shorter time to reach stable state; (3) the sooner the government disclosed information on the Internet, the sooner the public opinions reached stable state; (4) the more opinion leaders participated in the Internet public opinions, the earlier the opinions reached stable state.
\end{abstract}

Keywords: Computer simulation, emergence events, internet public opinion, opinion evolution.

\section{INTRODUCTION}

With the rapid development of the Internet and computer technology over the last decade, especially the rapid growth of population of netizens, the Internet has become an important vehicle and venue for information access and acquisition, which has an increasing effect on government's policy making, policy implementation, and governance. What the netizens voiced about their views, beliefs, and attitudes form the Internet public opinion. Compared with the traditional public opinion, the biggest different of Internet public opinion is online: it is formed online, spreads online, evolves online, and influences people online. The online nature makes the information, both real and unreal, propagate quickly, freely and widely; this may deeply influences people's ideas because there are so many people use the Internet today. In a sudden incident of major public concern such as the April, 2013 H7N9 bird flu outbreak, all kinds of information free flew on the Internet, among which one cannot tell the truth from the false. More than ten provinces were influenced in the event, and hundreds of people were infected among which 37 people were died. At the time of the outbreak, Chinese people were arguing online about if Banlangen, a herb in the traditional Chinese medicine, was able to prevent the H7N9 bird flu. Some people believed that Banlangen can prevent the bird flu and they stockpiled large amount of Banlangen. While others criticized online that it was a big lie from the medicine sellers and manufacturers just for more money. The remainder held a neutral attitude. Many heavily panic people ran into the medicine stores crazily and bought up all the Banlangen even in a high price.
The public should be told the real information in the crisis. If the government could do something to guild the Internet opinion properly, all those would not happen. This paper focused on the public concerned event -"whether Banlangen can prevent the H7N9 bird flu" as a case study to explore the evolution of public opinion on Internet.

\section{RELATED WORKS}

Recently, Chinese social networking sites (SNS) such as Weibo - "microblog", which is China's equivalence to and substitute for Twitter - are emerging as new social media, that are playing an increasingly important role in spreading public opinion, which deeply affects politics, economy, technology and culture [1]. Therefore, studying the propagation laws of Internet opinion on the Weibo platform has important values both in theory and in practice.

Research on evolution of public opinion has been a hot topic [2-6]. Some famous models have been developed, such as the Krause-Hegselmann model [7], Sznajd model [8], and Deffuant model [9]. In these models, an individual's opinion is based on his/her neighbors' views. On the other hand, public opinion, especially Internet public opinion, is a kind of dynamic collective attitude formed by numerous individuals. In this opinion, public opinion belongs to the area of emergent computation, which is an idea exhibited by multi-agent systems, and describes the process of enormous simple agents forming complex behaviors by cooperating [10]. However, at present few scholars have studied public opinions from this point of view, and in the works of those who did, the hypotheses are too idealistic and therefore lack practical applicability. For instance, with ideas of emergent computing, Wu et al. [5] studied the evolution process of public opinion, but did not consider the factors such as individuals' emotion; so their work could not repeat the evolution process 
of public opinion in real situations. Besides, in virtual environment, the individual's psychology and emotion also have an effect on his cognition and thinking, eventually influencing the final trend of public opinion. Therefore, someone proposed the concept of "emotional opinion in Internet" and analyzed its causes and guiding strategy. Later, some scholars did some researches on emotional Internet public opinion, but mostly from the perspective of social science. Also, there are some researchers who think about the emotional tendencies in public opinion, but the influence of emotion on the public opinion is not analyzed quantitatively. Based on above background, combined with the idea of Agent-Based Modeling and Simulation, some factors' impacts on the evolution are analyzed in our experiments.

\section{MODEL BUILDING FOR THE INFORMATION SPREADING IN EMERGENT EVENT}

\subsection{Conceptual Model}

The evolution of Internet public opinion is complex [4, 8]; so we will employ a few major factors for the model to be more manageable and to be sufficiently clear. Here a simplified model of influencing mode in emergent event has been built (Fig. 1).

Three components are in this model: government, netizens and crisis information. Different netizens have different influences; compared to the opinion leaders, common netizens have a smaller influence on others. Based on the influence, the netizens are separated into three parts: netizens with large/medium/small influence. The government can intervene in the Internet public opinion by announce some information to clarify the situation. To simplify the situation, the government is supposed to be an independent subject: it can influence netizens but cannot be influenced by netizens (The government is also influenced by netizens, but here we just discuss the influence from the government to netizens. In fact this is more likely in China's situations).

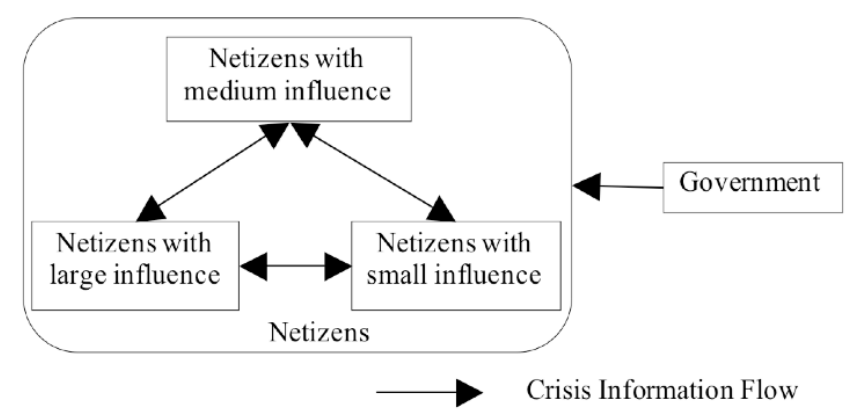

Fig. (1). Simplified model of influencing mode in emergent event.

\subsection{Properties of the Subjects}

The properties of the three components in our model are showed in Table $\mathbf{1}$.

Where,

- Attitude: The variable Attitude can be one of the three values: Approval, Opposition, and Neutrality. Here, approval means "I think Banlangen could prevent the bird flu H7N9", opposition means "I do not think Banlangen could prevent the bird flu H7N9" and neutrality means "I have no idea about whether Banlangen could prevent the bird flu H7N9 or not". $A_{i}(t)$ is the attitude of netizen $i$ at time t. When $A_{i}(t) \in[-1,-0.33]$, the attitude is approval; $\mathrm{A}_{\mathrm{i}}(\mathrm{t}) \in(-0.33,0.33]$ means neutrality; $\mathrm{A}_{\mathrm{i}}(\mathrm{t}) \in(0.33,1]$ means opposition.

- Type: Netizens in the model are classified as netizens, opinion leaders and network media, which is a system created by the leading Sina Weibo, and having been adopted by all other major weibos of major Chinese portal sites. In our model, $T_{1}$ stands for certified enterprise users, $T_{2}$ stands for certified individual users and $T_{3}$ stands for uncertified individual users.

- Conformity: Conformity level is to measure how easy one is influenced by others. $F_{i}$ is the degree that netizen $i$ is influenced by others, $F_{i} \in[0,1]$. The larger $F_{i}$ is, the larger one will be influenced by others. In extreme cases such as $\mathrm{F}_{\mathrm{i}}=1$ or $\mathrm{F}_{\mathrm{i}}=0$, one has no his/her own opinion or will never change his/ her opinion.

- Influence: Contrary to Conformity, Influence here stands for how strongly one can influence others. In the model, there are three different kinds of archetypes: large influence with $I_{i} \in[0,0.7]$, medium influence with $I_{i} \in(0.7$, $0.9]$ and small influence with $\mathrm{I}_{i} \in(0.9,1]$.

- Tend to Refute: $R_{k}(t)$ stands for the tendency of government refutes the information at time $t i$. The value is between -1 and $+1:-1$ stands for government refutes the information while 1 is the opposite. The closer $R_{k}(t)$ is to 1 , the more likely the government tends to refute the information.

- Credibility: $\mathrm{C}$ stands for the Credibility of government: how much the public believe the government. The value is between 0 and 1: the larger $\mathrm{C}$ is, the more the public believe the government.

- Rapidity: The Rapidity of information publication (V) is to measure the speed of the government information discloses. The value of $\mathrm{V}$ is between 0 and 1 : the closer $\mathrm{V}$ is to 1 , the more quickly the government refutes the information.

Table 1. Properties of the subjects.

\begin{tabular}{|c|c|c|c|}
\hline & Netizen (i) & Government (k) & Crisis Information \\
\hline \hline \multirow{3}{*}{ Properties } & Attitude: $\mathrm{A}_{\mathrm{i}}(\mathrm{t})$ & Tend: $\mathrm{R}_{\mathrm{k}}(\mathrm{t})$ & Importance $: \mathrm{P}$ \\
& Type: $\mathrm{T}_{\mathrm{i}}$ & Credibility: $\mathrm{C}$ & Ambiguity: $M(t)$ \\
& Conformity: $\mathrm{F}_{\mathrm{i}}$ & Rapidity: $\mathrm{V}$ & Intensity: $\mathrm{S}(\mathrm{t})$ \\
& Influence: $\mathrm{I}_{\mathrm{i}}$ & & \\
\hline
\end{tabular}


- Importance: Whether the crisis information is easy to spread is based on the Importance of the information to the netizens. The more important the information is, the easier it will be spread. P stands for the Importance and it is between 0 and 1: the larger the $\mathrm{P}$ value, the more important the information is to netizens.

- Ambiguity: Ambiguity is to measure how far the information to the real fact. The ambiguity will decrease with the information publicized. $M(t)$ stands for the ambiguity of the information at time $t$ and it is between 0 and 1: the larger the $M(t)$ value, the more unclear the information is to netizens.

- Intensity: Intensity of spread is an environment variable to describe the crisis information dissemination. $\mathrm{S}(\mathrm{t})$ stands for the Intensity of the information at time $t$ and $\mathrm{S}(\mathrm{t})=\mathrm{P} \times \mathrm{M}(\mathrm{t})$, and the value is between 0 and 1: the larger the $\mathrm{S}(\mathrm{t})$ value, the more intense the information is to netizens.

\subsection{Interaction Rules between Subjects}

Some parameters and relationship functions are set in this part.

In the Internet public opinion, the influence of netizen $i$ to netizen $\mathrm{j}$ at time $\mathrm{t}$ is $\mathrm{f}(\mathrm{i}, \mathrm{j}, \mathrm{t})$. As three different kinds of netizens are in the model (netizens with large/medium/small influence), three different functions are set:

$\mathrm{f}_{1}(\mathrm{i}, \mathrm{j}, \mathrm{t})=0.4 \mathrm{I}_{\mathrm{i}}(\mathrm{t})+0.2 \mathrm{~F}_{\mathrm{j}}(\mathrm{t})+0.4 \mathrm{~S}(\mathrm{t})$
$\mathrm{f}_{2}(\mathrm{i}, \mathrm{j}, \mathrm{t})=0.3 \mathrm{I}_{\mathrm{i}}(\mathrm{t})+0.3 \mathrm{~F}_{\mathrm{j}}(\mathrm{t})+0.4 \mathrm{~S}(\mathrm{t})$
$\mathrm{f}_{3}(\mathrm{i}, \mathrm{j}, \mathrm{t})=0.2 \mathrm{I}_{\mathrm{i}}(\mathrm{t})+0.5 \mathrm{~F}_{\mathrm{j}}(\mathrm{t})+0.4 \mathrm{~S}(\mathrm{t})$

where,

$\mathrm{I}_{\mathrm{i}}(\mathrm{t})$-- the Influence of information sender $\mathrm{i}$;

$F_{j}(t)$-- the Conformity of information receiver $j$;

$S(t)$-- the Intensity of the information.

The coefficients in the function 1.0 to 1.3 are referenced from Liang (2009) [11]. zens:

And $f(G, i, t)$ is the influence of the government to neti-

$\mathrm{f}(\mathrm{G}, \mathrm{i}, \mathrm{t})=0.4 \mathrm{~F}_{\mathrm{i}}(\mathrm{t})+0.4 \mathrm{C}+0.2 \mathrm{~V}$

where,

$F_{i}(t)$-- the conformity of information receiver $i$;

C -- the Credibility of the government;

$\mathrm{V}$-- the Rapidity of information publication.

If the difference of attitudes between sender $\mathrm{i}$ and receiver $\mathrm{j}$ is no more than $0.5\left(\left|\mathrm{~A}_{\mathrm{i}}(\mathrm{t})-\mathrm{A}_{\mathrm{j}}(\mathrm{t})\right|<=0.5\right)$, $\mathrm{i}$ and $\mathrm{j}$ are similar. At this time, receiver $\mathrm{j}$ will be influenced by sender I, Else, sender $i$ and receiver $j$ are quite different on attitude and the attitude of $\mathrm{j}$ will be the same at time $\mathrm{t}+1$ :

$$
\begin{aligned}
& A_{j}(t+1)=A_{j}(t)+\left(A_{i}(t)-A_{j}(t)\right) \times f(i, j, t) \text { if }\left|A_{i}(t)-A_{j}(t)\right|<=0.5 \\
& A_{j}(t+1)=A_{j}(t) \text { if }\left|A_{i}(t)-A_{j}(t)\right|>0.5
\end{aligned}
$$

The attitude of a netizen will also be influenced by the government:
$A_{i}(t+1)=A_{i}(t)+R_{k}(t) \times f(G, i, t)$

where $R_{k}(t)$ is the tendency of the government refute the information.

As time goes by, more and more information is publicized from the government. So netizens will know the event more and more clear and the tendency of the government refute the information will decrease:

$\mathrm{R}_{\mathrm{k}}(\mathrm{t}+1)=\mathrm{R}_{\mathrm{k}}(\mathrm{t})-\mathrm{A}_{\mathrm{i}}(\mathrm{t}) \times \mathrm{S}(\mathrm{t})$

Similarly, as more and more information is publicized from the government, Ambiguity and Intensity of the crisis information will decrease from time $t$ to $t+1$ :

$\mathrm{M}(\mathrm{t}+1)=\mathrm{M}(\mathrm{t})-\mathrm{V} \times \mathrm{f}(\mathrm{G}, \mathrm{i}, \mathrm{t})$

$\mathrm{S}(\mathrm{t}+1)=\mathrm{S}(\mathrm{t})-\mathrm{P} \times \mathrm{V} \times \mathrm{f}(\mathrm{G}, \mathrm{i}, \mathrm{t})$

\section{SIMULATIONS AND RESULTS}

\subsection{Data Collection}

The data were collected from a social networking site called Sina Weibo. Millions of Weibos were collected that contain "Banlangen" and "H7N9" between April 2 and April 5,2013 . We only took a small part of the data for our study.

\subsection{Initial Value of Parameters}

Before simulation, the initial value of parameters were defined below:

(1) Initial Attitude: Computing the number of the three kinds of Weibos (approval: n1, opposition: n2, neutrality: n3) of each netizen from the collected Weibos to find his/her main attitude (If $\mathrm{n} 1$ is larger than $\mathrm{n} 2$ and $\mathrm{n} 3$, then the netizen's attitude is approval). Here, $28 \%$ of netizens' attitude values Approval; $15 \%$ of netizens' attitude values Opposition; $57 \%$ of netizens' attitude values Neutrality. Based on the proportion randomly assigned the agents' attitude values to intervals $[-1,-0.33],(-0.33,0.33]$ and $(0.33,1]$.

(2) Initial Influence: Computing the number of each netizen's Weibo forwarded by others. If the number is more than 300 , he/she is a netizen with large influence. If it is between 50 and 300 , he/she is a netizen with medium influence. If it is less than 50 , he/she is a netizen with small influence. Here $60 \%$ of netizens are in interval [0, 0.7] (small influence); $29 \%$ of netizens are in interval $(0.7,0.9]$ (medium influence); $50 \%$ of netizens are in interval $(0.9$, 1](large influence). Based on the proportion, randomly assigned the agents' Influence values to intervals [0, 0.7], $(0.7$, $0.9]$ and $(0.9,1]$.

(3) Initial Conformity: Computing the number of forwarding Weibos (X) and supported forwarding Weibos (x) of a netizen. Dividing $\mathrm{x}$ by $\mathrm{X}$ is one's initial conformity $\left(\mathrm{F}_{\mathrm{i}}\right)$. Here $22 \%$ of netizens are in interval $[0,0.3], 28 \%$ of netizens are in interval $(0.3,0.7]$ and $50 \%$ of netizens are in interval $(0.7,1]$. Based on the proportion, randomly assigned the agents' Conformity values to intervals $[0,0.3],(0.3,0.7]$ and $(0.7,1]$.

(4) Initial Type: Computing the number of the three kinds of netizens (certified enterprise users, certified individual users and uncertified individual users). Here, $10 \%$ of netizens are certified enterprise users $\left(\mathrm{T}_{1}=0.1\right) ; 20 \%$ of neti- 


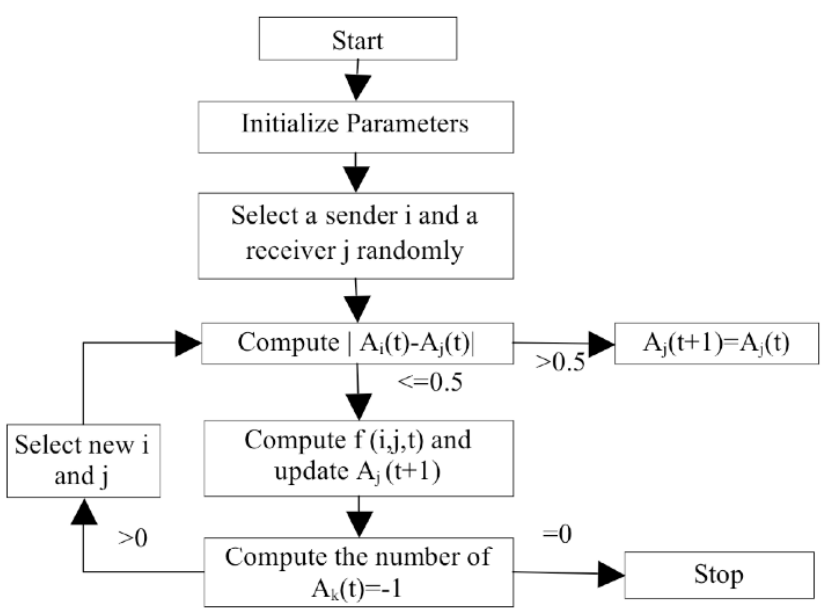

Fig. (2). Algorithms between netizen agents.

zens are certified individual users $\left(\mathrm{T}_{2}=0.2\right)$; and $70 \%$ of netizens are uncertified individual users $\left(\mathrm{T}_{3}=0.7\right)$. Based on the proportion, randomly assigned the agents' type values to $T_{1}$, $\mathrm{T}_{2}$ and $\mathrm{T}_{3}$.

(5) Tendency of Government Refutation: Computing the number of Weibos with refuting information $\left(\mathrm{y}_{1}\right)$ and Weibos without refuting information $\left(\mathrm{y}_{2}\right) \cdot \mathrm{R}\left(\mathrm{t}_{0}\right)=\left(\mathrm{y}_{1}-\mathrm{y}_{2}\right) /\left(\mathrm{y}_{1}+\right.$ $\mathrm{y}_{2}$ ), and the closer $\mathrm{R}\left(\mathrm{t}_{0}\right)$ is to 1 , the more likely the government tend to refute the information. Here the value of $R_{0}(t)$ is -0.46 , which was got from the real data.

(6) Initial Intensity It is not clear the intensity of the crises information, here we suppose the Intensity of the crises information at time $\mathrm{t}_{0}$ is $0.5\left(\mathrm{~S}\left(\mathrm{t}_{0}\right)=0.5\right)$.

\subsection{Algorithm Description}

Figs. (2) and (3) show the algorithms of the study.

\subsection{Hypotheses}

Four hypotheses have been posited before the simulations:

H1: The Internet public opinion will have a shorter time to reach stable state with government interventions than without government interventions.

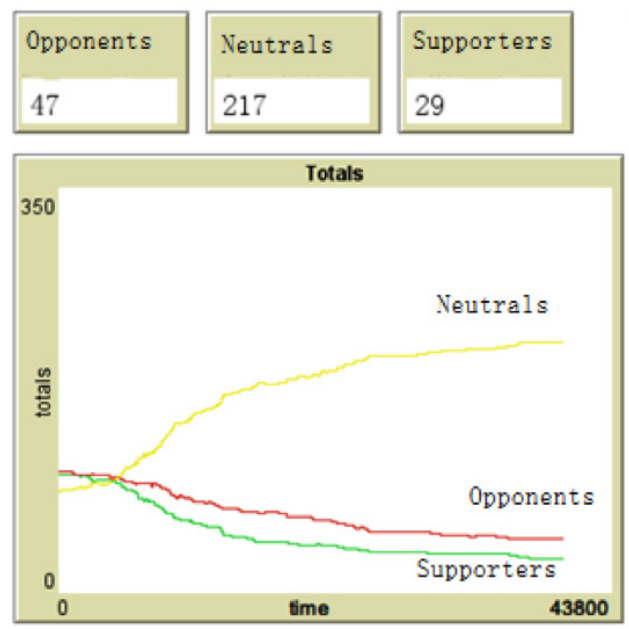

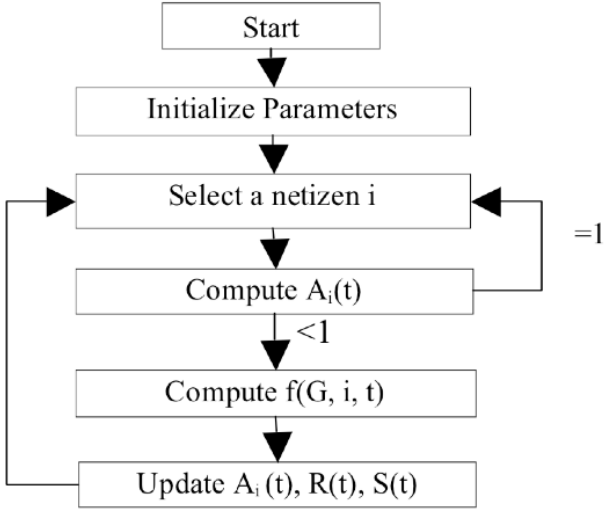

Fig. (3). Algorithms between netizen agents and government agents.

H2: The Internet public opinion will have a shorter time to reach stable state with a higher government's credibility.

H3: The more rapidly the government information disclose in the Internet public opinion the earlier it will reach stable state.

H4: The more opinion leaders in the Internet public opinion the earlier it will reach stable state.

\subsection{Simulation Results}

In order to verify the correctness of the hypotheses put forward before, the parameters are changed and the results are in the following figures, in which green lines mean the number of approving netizens, purple lines mean the number of disapproving netizens, and yellow lines mean the number of neutral netizens.

(1) Keeping other conditions the same, one situation is without government interventions and another is with government interventions.

From Fig. (4) we can see that it has a shorter time with government interventions than without government interventions to reach stable state. Without government interventions most people tend to be neutral while with government interventions most people tend to be approved. So, the first hypothesis is supported.
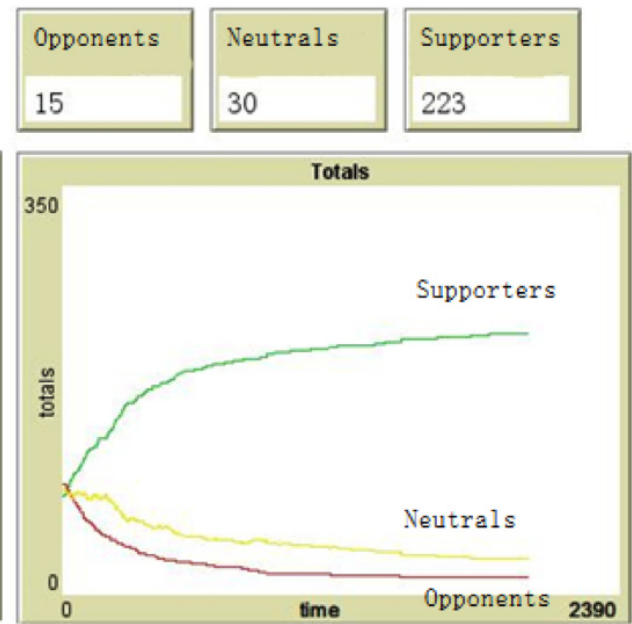

Fig. (4). View of the situation of without government interventions vs. with government interventions. 

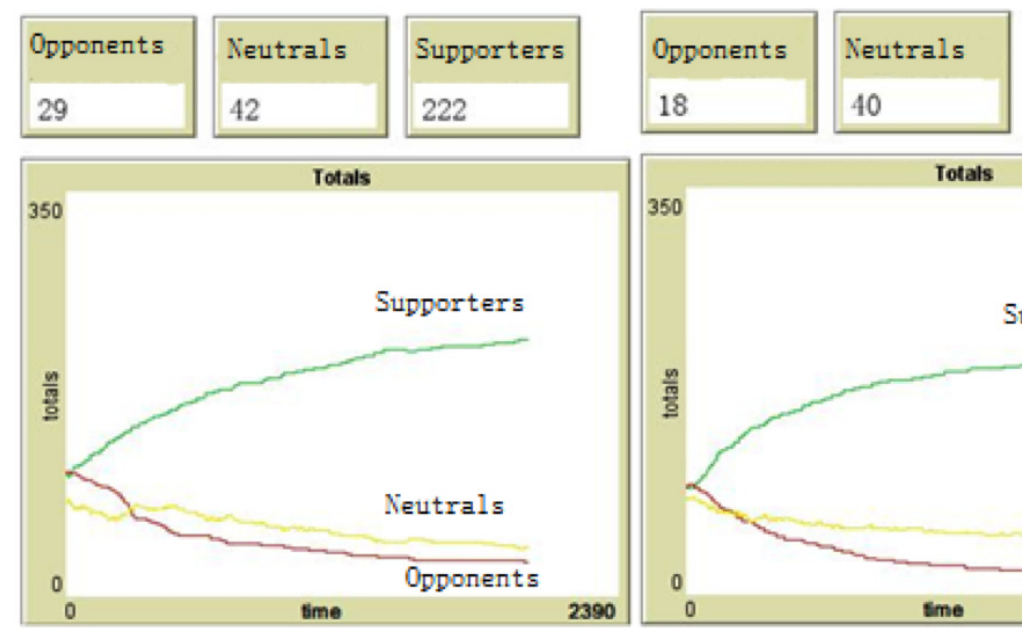

Supporters

210

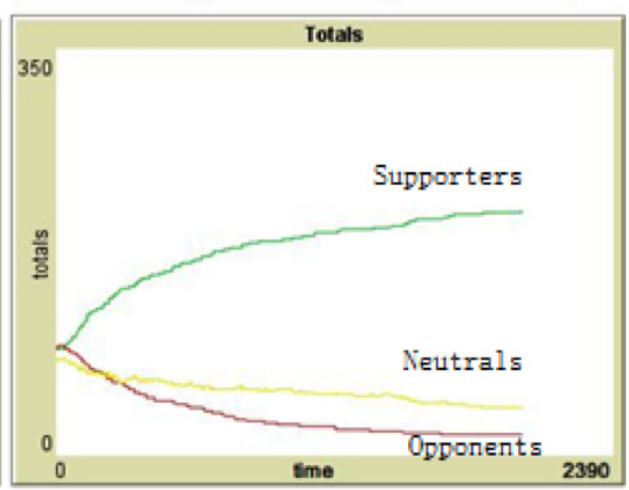

Fig. (5). View of the situation of small government credibility vs. large government credibility.
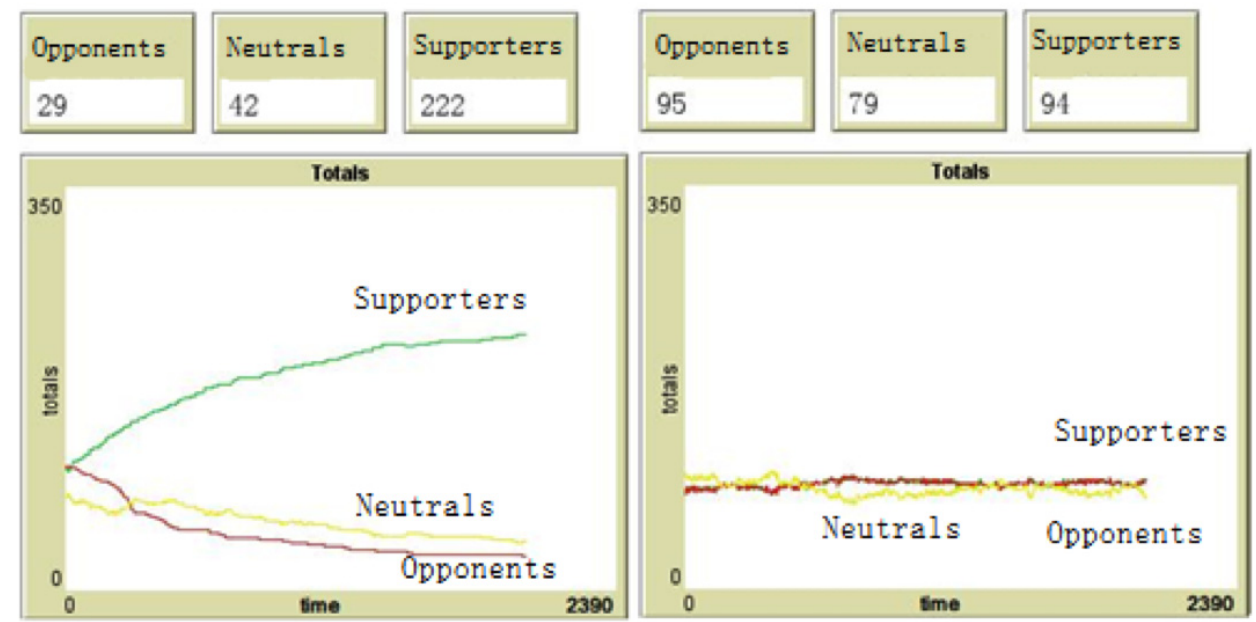

Fig. (6). View of the situation of disclosing the government information slowly vs. quickly.
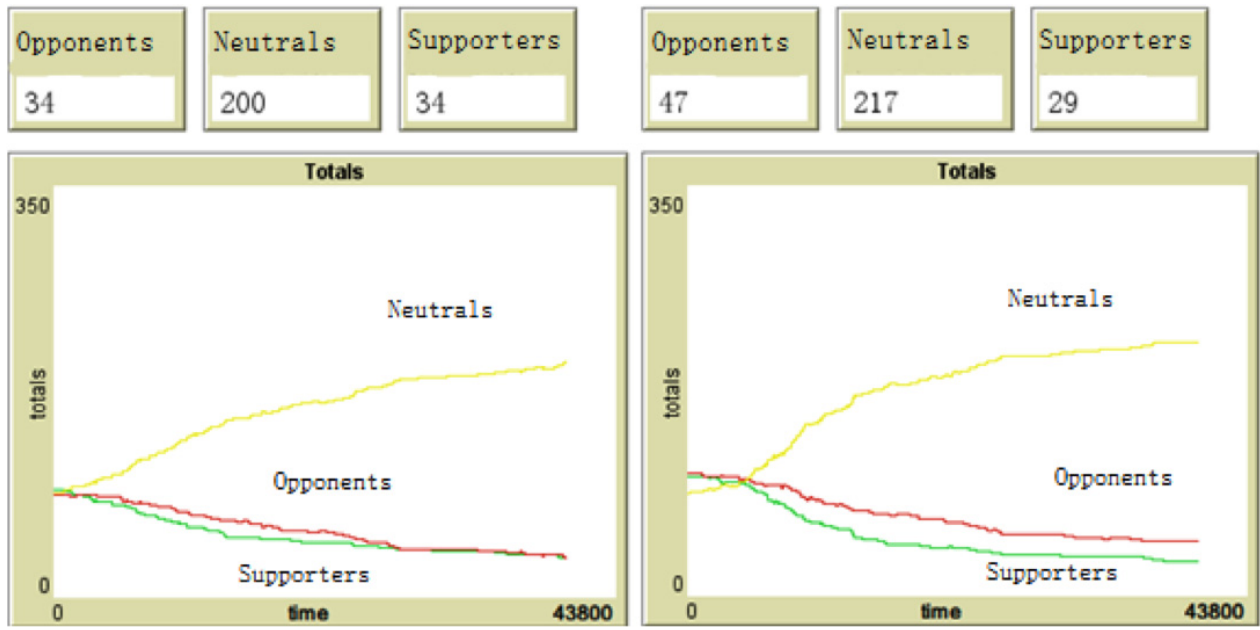

Fig. (7). View of the situation of few opinion leaders vs. many opinion leaders.

(2) Keeping other conditions the same, one situation is with small government credibility and another is with large government credibility.

From Fig. (5) we can see that it has a shorter time with large government credibility than small government credibility to reach stable state. So, the second hypothesis is supported.
(3) Keeping other conditions the same, one situation is with slowly disclosing the government information and another is with quickly disclosing the government information.

From Fig. (6) we can see that the more rapidly the government information disclosed in the Internet public opinion the earlier it will reach stable state. So, the third hypothesis is supported. Here an interesting result is that netizens' atti- 
tudes distribute very equally with quickly disclosing the government information. It deserves our further research.

(4) Keeping other conditions the same, one situation is with few large influenced netizens and another is with many large influenced netizens.

From Fig. (7) we can see that the more opinion leaders in the Internet public opinion the earlier it will reach stable status. So, the forth hypothesis is supported.

\section{CONCLUSION}

In this study, we conducted a simulation using NetLogo, on the interactions among three players of China's SNS Weibo (micro-blog) regarding the forming of public opinions. From the results of the simulation, we can see that the government plays an important role in the forming of Internet public opinion in China: Firstly, government interventions can make the Internet public opinion reach stable status more quickly. Specifically and interestingly, in scenarios without government interventions, most people tend to be neutral in their opinions, while with government interventions most people's opinions tend to be approval. Normally the majority of netizens are neutral because they are not positive about the arguments of the two extremes; but when the government issues information that is approval in tone, most netizens accepted the government's information and became approval too. Secondly, when the government has high credibility and provides rapid information disclosure, the time for the public opinions to reach stable state will be short. The government should create a fair and open Internet environment and keep the information flow freely on the Internet that everyone could know the truth, in first place. An interesting result is that netizens' attitudes distribute very equally with rapid information disclosure. Finally, leveraging the opinion leaders can have a good effect on the Internet public opinion.

\section{CONFLICT OF INTEREST}

The authors confirm that this article content has no conflict of interest.

\section{ACKNOWLEDGEMENTS}

This work was financially supported by the National Natural Science Foundation of China (71273132).

\section{REFERENCES}

[1] X. M. Si, Y. Liu, F. Ding and F. Xiong, "Research on bounded confidence consensus emergency model with community structure", Journal of System Simulation, vol. 21, pp. 7644-7647, 2009.

[2] C.Y. Liu, X.F. Hu, P. Luo, and G.Y. Si, "Study on agent-based communication network model of public opinion on internet", Computer Simulation, vol. 26, pp. 20-23, 2009.

[3] J. Song, L. R. Gan, P. Wu, and J. Zhao, "Dynamics evolution and simulation of internet public opinion: considering netizens' preference for polarity of opinion," Geomatics and Information Science of Wuhan University, vol. 39, pp. 223-229, 2014.

[4] M.H. He, D.M. Zhang, H.Y. Wang, and X.G. Li, "Public opinion evolution model with the variable topology structure based on scale free network", Acta Physica Sinica, vol. 59, pp. 5175-5181, 2010.

[5] H. Wu, and M. Xin, "A quick emergency response model for micro-blog public opinion crisis oriented to mobile internet services: design and implementation", Journal of Software, vol. 7, pp. 1413$1420,2012$.

[6] F. J. Chen and L. B. Li, "Application of G (Galam) model in network public opinion evolution", Journal of Computer Applications, vol. 31, pp. 3411-3413, 2011.

[7] S. Fortunato, "The Krause-Hegselmann consensus model with discrete opinions", International Journal of Modern Physics C, vol. 15, pp. 1021-1029, 2004.

[8] K. Sznajd-Weron, and J. Sznajd, "Opinion evolution in closed community”, International Journal of Modern Physics C, vol. 11, pp.1157-1165, 2000.

[9] G. Deffuant, D. Neau, F. Amblard, and G. Weisbuch, "Mixing beliefs among interacting agents", Advances in Complex Systems, vol. 3, pp. 87-98, 2000.

[10] J. Li, X. G. Zhou, and B. Chen, "Research on analysis and monitoring of internet public opinion", Advances in Intelligent Systems and Computing. vol. 191, pp. 449-453, 2013.

[11] H. X. Liang, "Research on simulation of the crisis information communication on the internet", MS thesis, Harbin Engineering University, Harbin, ON, China, 2011.

Received: June 10, 2015

(C) Jiang and Peng; Licensee Bentham Open.

This is an open access article licensed under the terms of the Creative Commons Attribution Non-Commercial License (http://creativecommons.org/licenses/by-nc/3.0/) which permits unrestricted, non-commercial use, distribution and reproduction in any medium, provided the work is properly cited. 